\title{
RUNGE-KUTTA DISCONTINUOUS GALERKIN METHOD FOR HYPERBOLIC HYPERELASTICITY EQUATIONS FOR INHOMOGENEOUS MEDIUM
}

\author{
M.V. ALEKSEEV ${ }^{*}$, E.B. SAVENKOV \\ Keldysh Institute of Applied Mathematics, Russian Academy of Sciences. Moscow, Russia \\ *Corresponding author. E-mail: mikhail.alekseev@phystech.edu
}

DOI: $10.20948 /$ mathmontis-2020-47-5

Summary. The work considers application of Runge-Kutta Discontinuous Galerkin method for solution of Godunov-Romenskii type hyperbolic model for hyperelastic medium. The medium is considered inhomogeneous with piecewise uniform distributed properties. To describe evolution of medium Godunov-Romenskii model is used supplemented with transport equation that describes evolution of properties distribution. The numerical approach is based on application of Runge-Kutta Discontinuous Galerkin method with Godunov type fluxes both for conservative and non-conservative terms. We describe mathematical model and corresponding numerical algorithm briefly. Results of numerical simulations are presented.

\section{INTRODUCTION}

The present paper is devoted to numerical study of Runge-Kutta Discontinuous Galerkin (RK/DG) method of high order of accuracy for solution of first order hyperbolic system of equations of hyperelasticity.The model describes dynamics of continuous media (deformation and strain fields, velocity, temperature and entropy) in Eulerian reference frame. The model was originally proposed[1] by S.Godunov and E.Romenskii. Recently there has been a significant increase in interest in such type of the models since it is assumed that in some cases they are more suitable (comparably to traditional models based on arbitrary Lagrangian-Eulerian description) to simulation of physical phenomena involving extremely large deformations of the media[2,3]. Such type of problems are often arise in numerical simulation of shock wave phenomena in solids induced by rapid mechanical, thermal or radiation loads[24, 25].

Currently, a number of papers is devoted to numerical solution of hyperelasticity model[4, 5]. However, the most of them considers WENO-based approaches[6, 7]. In present work Runge-Kutta Discontinuous Galerkin method[8] is considered. The general motivation for such a choice is its universality and possibility of generalization to higher-order equations, that may occur in the multiphase problems. The second reason is to estimate efficiency of the RK/DG method when simulating Godunov-Romenskii model for particular cases of more simple (gas and fluid dynamics) and more complex (inhomogenious hyperelastic medium) settings. Both issues can be considered as a preliminary tests for further development of RK/DG numerical techniques for complex multiphase and multicomponent models developed in, e.g., Baer-Nunziato framework[9].

The main features of present paper are: 
- first order hyperbolic hyperelastic model is considered as a unified framework to describe solid/liquid/gaseous media;

- heterogeneous elastic medium case which assumes piecewise uniform distribution of medium properties is considered .

The structure of the paper is as follows. The basic Godunov-Romenskii hyperelastic model is described at the beginning of the section 2. In the subsection 2.1 its simple generalization to the case of piecewise homogeneous case is considered. Section 3 is devoted to the description of the RK/DG numerical algorithm for both conservative and non-conservative hyperbolic equations. In section 4 the implementation detalis and results of numerical experiments are presented.

\section{MATHEMATICAL MODEL}

To describe dynamics of the continuous hyperelastic medium in the Euler reference frame the Godunov-Romenskii model[10] is used. The corresponding system of equations is hyperbolic and consists of conservation law of momentum (1), dynamic equations for distortion tensor components (2) and conservation law of energy (3):

$$
\begin{gathered}
\frac{\partial(\rho \mathbf{u})}{\partial t}+\nabla \cdot(\rho \mathbf{u} \otimes \mathbf{u}-\mathbf{T})=0 \\
\frac{\partial(\rho \mathbf{F})}{\partial t}+\nabla \cdot\left(\rho \mathbf{F} \otimes \mathbf{u}-\rho \mathbf{u} \otimes \mathbf{F}^{T}\right)=-\mathbf{u} \otimes \nabla \cdot(\rho \mathbf{F}), \\
\frac{\partial(\rho E)}{\partial t}+\nabla \cdot(\rho \mathbf{u} E-\mathbf{u} \otimes \mathbf{T})=0 .
\end{gathered}
$$

Here $\mathbf{T}$ is Cauchy stress tensor, $E=\mathrm{U}+|\mathbf{u}|^{2} / 2-$ total energy, $\mathrm{U}=\mathrm{U}(\mathbf{F}, \mathrm{S})-$ internal energy. The primary variables are components of distortion tensor $\mathbf{F}=\nabla_{\mathbf{X}} \mathbf{x}$ ( $\mathbf{x}$ and $\mathbf{X}$ are Euler and Lagrange coordinates of medium points, respectively), velocity $\mathbf{u}$ and entropy $\mathbf{S}$. Symbol " $\otimes$ " denotes the tensor product. The medium density $\rho$ is defined as

$$
\rho=\rho_{0} / \operatorname{det}(\mathbf{F}),
$$

where $\rho_{0}=\rho_{0}(\mathbf{x})$ denotes the density of undeformed medium. The combination of equations (2) and (4) recovers the continuity equation:

$$
\frac{\partial \rho}{\partial t}+\nabla \cdot(\rho \mathbf{u})=0
$$

This equation can be used instead of one of the equations in (2) for the distortion tensor components.

System (1)-(3) has to be closed by the specific internal energy (equation of state, EOS) in its canonical form, $U=U(F, S)$. To provide the frame indifference of internal energy it must be expressed in terms of some symmetric strain tensor $\mathbf{G}[10,5]$ : 


$$
\mathrm{U}=\mathrm{U}(\mathbf{F}, \mathrm{S})=\hat{\mathrm{U}}(\mathbf{G}, \mathrm{S}) \text {. }
$$

Here and further $\mathrm{U}, \hat{\mathrm{U}}, \mathrm{U}^{+}, \ldots$ denote functional dependencies of the same variable on particular set of arguments.

A number of strain tensors $[10,11]$ can be used in (5). In the present work the Finger tensor $\mathbf{G}=\mathbf{F}^{-T} \mathbf{F}^{-1}$ is considered[4]. In this case the Cauchy stress tensor $\mathbf{T}$ is expressed by Murnaghan formula[10]:

$$
\mathbf{T}=-2 \rho \mathbf{G} \cdot \frac{\partial \hat{\mathbf{U}}}{\partial \mathbf{G}}
$$

Since $\hat{U}$ is a function of Finger tensor components it can be expressed, due to objectivity arguments, as a function of its invariants $I_{1,2,3}$ :

$$
\begin{gathered}
\overline{\mathrm{U}}=\mathrm{U}^{+}\left(I_{1}, I_{2}, I_{3}, \mathrm{~S}\right), \\
I_{1}=\operatorname{tr}(\mathbf{G}), I_{2}=\left[\operatorname{tr}(\mathbf{G})^{2}-\operatorname{tr}\left(\mathbf{G}^{2}\right)\right] / 2, I_{3}=\operatorname{det}(\mathbf{G}) .
\end{gathered}
$$

The internal energy $\bar{U}$ can be considered as the sum of two terms. The first one, $U^{\text {h }}$, is "hydrodynamical" part that depends only on bulk deformation and the second one, $\mathrm{U}^{\mathrm{sh}}$, describes dependency on shear deformation:

$$
\mathrm{U}^{+}=\mathrm{U}^{\mathrm{h}}\left(I_{3}, \mathrm{~S}\right)+\mathrm{U}^{\mathrm{sh}}\left(I_{1}, I_{2}, I_{3}, \mathrm{~S}\right) .
$$

Hereafter the isotropic hyperelastic EOS[4] is used:

$$
\begin{gathered}
\mathrm{U}^{\mathrm{h}}\left(I_{3}, \mathrm{~S}\right)=\frac{K_{0}}{2 \alpha^{2}}\left(I_{3}^{\alpha / 2}-1\right)^{2}+c_{V} T_{0} I_{3}^{\gamma / 2}\left(\exp \left[\mathrm{S} / c_{V}\right]-1\right), \\
\mathrm{U}^{\mathrm{sh}}\left(I_{1}, I_{2}, I_{3}\right)=B_{0} I_{3}^{\beta / 2}\left(I_{1}^{2} / 3-I_{2}\right) / 2 .
\end{gathered}
$$

Here $K_{0}=c_{0}^{2}-(4 / 3) b_{0}^{2}$ is the squared bulk sound velocity, $c_{0}$ is sound velocity, $b_{0}$ is shear elastic wave velocity, $B_{0}=b_{0}^{2}, c_{V}$ is the volumetric heat capacity, $T_{0}$ is reference temperature, $\alpha, \beta, \gamma$ are constant parameters.

The considered model can describe both solids $\left(\partial \mathrm{U}^{+} / \partial I_{1,2,3} \not \equiv\right)$ and liquids/gases $\left.\partial \mathrm{U}^{+} / \partial I_{1,2} \equiv 0, \partial \mathrm{U}^{+} / \partial I_{3} \not \neq 0\right)$. In the latter case the system of equations (1)-(3) can be reduced to classical gas dynamics equations with only bulk deformation accounted. That can be done by replacing the equations for distortion tensor components (2) with mass conservation law.

\subsection{Piecewise homogeneous model}

Consider now spatial domain $\Omega$ occupied by piecewise homogeneous medium. The latter means that parameters of EOS (9), (10) are different in different subdomains (phases) of $\Omega$. 
Let $\Omega_{k}, k=\overline{1, N_{f}}$ where $N_{f}$ is number of phases, be such subdomains, $\Omega=\bigcup \Omega_{k}, k=\overline{1, N}_{f}$. Let $\phi_{k}=\phi_{k}(\mathbf{X})$ be characteristic functions of $\Omega_{k}$ :

$$
\phi_{k}(\mathbf{X})=\left\{\begin{array}{ll}
1, & \mathbf{X} \in \Omega_{\mathrm{k}}, \\
0, & \mathbf{X} \notin \Omega_{\mathrm{k}},
\end{array} \quad k=\overline{1, N_{f}} ; \quad \sum_{k=1}^{N_{f}} \phi_{k}(\mathbf{X})=1 .\right.
$$

Set of EOS parameters specific for subdomain $\Omega_{k}$ is defined as $\mathbf{a}^{(k)}=\left\{\alpha, \beta, \gamma, K_{0}, \ldots\right\}$, $k=\overline{1, N_{f}}$. The EOS in that case has the form $\mathrm{U}_{k}=\mathrm{U}_{k}\left(\mathbf{G}, \mathrm{S} ; \mathbf{a}^{(k)}\right)$ in domain $\Omega_{k}$. Then the distribution of medium property in the Lagrangian reference frame is defined as

$$
\mathbf{a}(\mathbf{X})=\sum_{k=1}^{N_{f}} \mathbf{a}^{(k)} \phi_{k}(\mathbf{X}) \text {. }
$$

During deformation of the medium, the values of characteristic functions $\hat{\phi}_{k}=\hat{\phi}_{k}(\mathbf{x}, t)$ in Eulerian reference frame satisfy the following equation:

$$
\frac{\partial \hat{\phi}_{k}}{\partial t}+\mathbf{u} \frac{\partial \hat{\phi}_{k}}{\partial \mathbf{x}}=0
$$

where $\mathbf{u}(\mathbf{x}, t)$ is velocity defined as a function of Eulerian coordinates. Equation (13) should be supplemented by appropriate initial conditions.

Further we do not use characteristic functions $\hat{\phi}_{k}$ but rather their "smoothed" version. The smoothed zone width is a parameter of the model and is resolved by the computational mesh used in simulations. Equation (13) is nonconservative and is solved together with hyperelasticity model equations (1)-(3).

Considered above inhomogeneous model assumes that only one EOS is used to describe behavior of all phases, - that is, it is not "real" multiphase model. However, it has a number of features of multiphase models: e.g., it consists of two groups of equations (conservative and nonconservative) $[12,9]$. In present work this model is considered as the simplest one to test and verify algorithmic techniques for numerical solution of more complicated models.

\section{NUMERICAL ALGORITHMS}

The considered class of problems is described by the hyperbolic system of equations of the first order. The total number of equations is large (13 equations of hyperelastic model plus $N_{f}$ equations for $\phi_{k}$ ), and its solution has a rich wave structure. The system consists of conservative and nonconservative equations. Its possible generalizations include spatial operators of higher order (for example, when considering surface tension). This motivates the following requirements for numerical methods:

- The method must be capable for calculations with high approximation accuracy.

- It has to provide possibility to construct numerical approximations of the hyperbolic operators as well as of diffusion ones.

- It can be applied in conservative and nonconservative settings. 
- It should provide unified framework when considering both theoretical and software implementation issues.

We consider RK/DG as a candidate for such a framework. Further we briefly describe it for both conservative and nonconservative cases.

Conservative case. Consider one-dimensional conservation law in spatial domain $\Omega=[0, L] \subset \mathrm{R}:$

$$
\frac{\partial g(x, t)}{\partial t}+\frac{\partial \mathrm{F}(g(x, t))}{\partial x}=0
$$

where $g(x, t)$ is conserved quantity and $\mathrm{F}(g)$ is corresponding physical flux.

Let $\left\{\omega_{i}\right\}_{i=0}^{i=N}$ be a partition of $\Omega$ into computational cells, and $\omega_{i}=\left[x_{i-1 / 2}, x_{i+1 / 2}\right], 1, i, N$. We shall denote by $V_{h}^{k}(\Omega)$ the space of elements of $L^{\infty}(\Omega)$ whose restriction to $\omega_{i}$ belongs to a vector space $\mathrm{P}^{k}\left(\omega_{i}\right)$ of polynomials of degree $k$ :

$$
\mathrm{V}_{h}^{k}=\left\{v:\left.v\right|_{\omega_{i}} \in \mathrm{P}^{k}\left(\omega_{i}\right) ; 1, i, N\right\} .
$$

Define the elements of $\mathrm{P}^{k}\left(\omega_{i}\right)$ by linearly independent orthogonal set of Legendre polynomials $\left\{\psi_{i}^{(l)}\right\}_{l=0}^{l=k}$ and replace exact solution $g(x, t)$ in $\omega_{i}$ by its approximation

$$
\left.g_{h}(x, t)\right|_{\omega_{i}}=\sum_{l=0}^{k} \psi_{i}^{(l)}(x) g_{i}^{(l)}(t)
$$

In order to obtain the semidiscrete equation for function $g_{h}(x, t)$ we multiply equation (14) by test function $v_{h} \in \mathrm{V}_{h}^{k}$, integrate over $\omega_{i}$ and apply Green's formula:

$$
\int_{\omega_{i}} \frac{\partial g_{h}(x, t)}{\partial t} v_{h}(x) d x+\int_{\omega_{i}} \mathrm{~F}\left(g_{h}(x, t)\right) \frac{\partial v_{h}(x)}{\partial x} d x+\sum_{e \in \partial \omega_{i}} \int_{j} \hat{\mathrm{F}}\left(g_{h}(x, t)\right) v_{h}(x) d \Gamma=0 .
$$

In equation (16) physical flux $\mathrm{F}\left(g_{h}(x, t)\right)$ is replaced by numerical flux $\hat{\mathrm{F}}\left(g_{h}(x, t)\right)$ in surface integral. In one-dimensional case one can obtain:

$$
\sum_{e \in \partial \omega_{i} e} \int_{\mathrm{F}} v_{h} d \Gamma=\hat{\mathrm{F}_{i+1 / 2}} v_{h}\left(x_{i+1 / 2}^{+}\right)-\hat{\mathrm{F}_{i-1 / 2}} v_{h}\left(x_{i-1 / 2}^{-}\right) .
$$

Here $\hat{\mathrm{F}}_{i \pm 1 / 2}$ is numerical flux at $x \in \partial \omega_{i}$.

$$
\hat{\mathrm{F}}_{i \pm 1 / 2}=\hat{\mathrm{F}}\left(g_{i \pm 1 / 2}^{+}, g_{i \pm 1 / 2}^{-}\right) \text {, }
$$

where $g_{i \pm 1 / 2}^{+}$and $g_{i \pm 1 / 2}^{-}$are left and right hand side limiting values of $g_{h}$ at $x \in \partial \omega_{i}$. Different numerical fluxes for hyperbolic hyperelastic models are known (including HLLC, HLL, etc.)[4]. We consider here only two options: 
1. Lax-Friedrichs flux:

$$
\hat{\mathrm{F}_{i \pm 1 / 2}^{\mathrm{LF}}}=\frac{1}{2}\left[\mathrm{~F}\left(g_{i \pm 1 / 2}^{+}\right)+\mathrm{F}\left(g_{i \pm 1 / 2}^{-}\right)\right]-\frac{1}{2} \frac{\Delta x}{\Delta t}\left(g_{i \pm 1 / 2}^{+}-g_{i \pm 1 / 2}^{-}\right) ;
$$

2. Rusanov flux:

$$
\hat{\mathrm{F}}_{i \pm 1 / 2}^{\mathrm{RS}}=\frac{1}{2}\left[\mathrm{~F}\left(g_{i \pm 1 / 2}^{+}\right)+\mathrm{F}\left(g_{i \pm 1 / 2}^{-}\right)\right]-\frac{1}{2} \Lambda\left(g_{i \pm 1 / 2}^{+}-g_{i \pm 1 / 2}^{-}\right),
$$

here

$$
\Lambda=\Lambda\left(g_{i \pm 1 / 2}^{+}, g_{i \pm 1 / 2}^{-}\right)=\max \left(\left|\lambda_{i \pm 1 / 2}^{+}\right|,\left|\lambda_{i \pm 1 / 2}^{-}\right|\right),
$$

where $\lambda_{i \pm 1 / 2}^{+}, \lambda_{i \pm 1 / 2}^{-}-$eigenvalues of Jacobian matrices $J\left(g_{i \pm 1 / 2}^{+}\right)$and $J\left(g_{i \pm 1 / 2}^{-}\right)$, $J(g)=\partial \mathrm{F}(g) / \partial g$.

Considering test functions in the form $v_{h}(x)=\left\{\psi_{i}^{(l)}\right\}_{l=0}^{l=k}$ the following system of ordinal differential equation is obtained for vector of coefficients $\hat{\mathbf{g}}=\left\{g_{i}^{(l)}\right\}_{l=0}^{l=k}$ :

$$
\frac{d \hat{\mathbf{g}}}{d t}=\mathbf{M}(\hat{\mathbf{g}})
$$

For time discretization of (17) a strong stability preserving TVD/RK3 method[8] is used. The appropriate limiting procedure (see below) is applied at each Runge-Kutta stage.

Nonconservative case. Let us describe now the RK/DG method applied to the nonconservative equation (13) in spatial domain $\Omega=[0, L] \subset \mathrm{R}$.

For (13) the traditional approach can not be applied in the same way as it was done previously for conservative case. The main cause is the difficulty of the definition of the solution $g(x, t)$ in terms of distributions. The correct formulation of the Riemann problem and corresponding generalized Hugoniot conditions can not be set in traditional way. The constructive solution to this problem is provided by DLM (DalMaso-LeFloch-Murat) theoretical framework[13].

Consider the discontinuous function

$$
g(x)=g_{1}+H\left(x-x_{\mathrm{d}}\right) g_{\mathrm{r}},
$$

where $H=H(x)$ is Heaviside function, $x_{\mathrm{d}}$ is the discontinuity coordinate, $g_{r, l}=g\left(x_{d} \pm 0\right)$ are the right and left-hand solution limits, respectively. For this case the expression

$$
a(x, g) \frac{\partial g}{\partial x},
$$


where function $a(x, g)$ has the discontinuity at the same point $x_{\mathrm{d}}$, can not be defined correctly as distirbution[14] and the special treatment is needed as developed in DLM theory. To proceed, replace $g$ by its smooth regularization $g_{\varepsilon}$ :

$$
g_{\varepsilon}(x)=\left\{\begin{array}{cc}
g_{1}, & x<x_{\mathrm{d}}-\varepsilon, \\
\Phi\left(\frac{x-x_{\mathrm{d}}+\varepsilon}{2 \varepsilon}\right), & x_{\mathrm{d}}-\varepsilon, x, x_{\mathrm{d}}+\varepsilon, \\
g_{\mathrm{r}}, & x>x_{\mathrm{d}}+\varepsilon,
\end{array}\right.
$$

where $\varepsilon>0$, mapping $\Phi:[0,1] \mapsto\left[g_{1}, g_{\mathrm{r}}\right]$ is Liepshitz continuous and is called path[13]. Now define non-conservative product (18) as

$$
a(x, g) \frac{\partial g}{\partial x}=\lim _{x \rightarrow x_{\mathrm{d}}} a\left(x, g_{\varepsilon}\right) \frac{\partial g_{\varepsilon}}{\partial x},
$$

In such a way at $\varepsilon \rightarrow 0$ the product (18) can be defined as bounded Borel measure, converging to $(18)$ in $*$-weak topology:

$$
a\left(g_{\varepsilon}\right) \frac{\partial g_{\varepsilon}}{\partial x} \rightarrow C \delta\left(x-x_{\mathrm{d}}\right), \quad C=\int_{0}^{1} a(\Phi(\tau)) \frac{\partial \Phi(\tau)}{\partial \tau} d \tau,
$$

where $\delta(x)$ is Dirac delta-function.

Consider again spatial domain $\Omega=[0, L] \subset \mathrm{R}$ with given partition $\left\{\omega_{i}\right\}_{i=0}^{i=N}$. Define the space of boundary points $\Gamma=\left\{x: x \in \partial \omega_{i}\right\}, i=\overline{1, N}$. Introduce the piecewise polynomials space $\mathrm{V}_{h}^{k}$ as was done previously. Multiply (13) by test function $v_{h} \in \mathrm{V}_{h}^{k}$ and integrate it over $\Omega$ taking (19) into account. This leads to the following semidiscrete equation for $g_{h} \in \mathrm{V}_{h}^{k}$ :

$$
\int_{\Omega} \frac{\partial g_{h}}{\partial t} v_{h} d x+\sum_{\omega_{i} \in \Omega_{\omega_{i}}} a\left(x, g_{h}\right) \frac{\partial g_{h}}{\partial x} v_{h} d x+\sum_{x \in \Gamma}\left\langle v_{h}\right\rangle_{x} \int_{0}^{1} \frac{\partial \Phi}{\partial \tau}(\tau) a(\Phi(\tau)) d \tau=0
$$

where $\left\langle v_{h}\right\rangle=\left(v_{h}^{\mathrm{r}}+v_{h}^{1}\right) / 2$. Borel measure (19) depends on the choice of the path $\Phi$. In the present work the linear path $\Phi(\tau)=\left(g_{\mathrm{r}}-g_{1}\right) \tau+g_{1}$ is chosen[13]. Further one can proceed as in the conservative setting.

Limiting procedure. For considered method the numerical solution will not be monotonic in case of discontinuous solution. To avoid non-physical oscillations in numerical solution an artificial dissipation has to be introduced. It can be done in various ways, among which methods based on geometric limiting, explicit introduction of additional dissipative terms and algorithms based on high-pass filtering component of the solution are known [15]. In [16] method for monotonizing the solution by explicitly introducing von Neumann-Richtmeier type artificial viscosity is described. The most popular technique is to use geometrical and moment-based limiters such as maximum preserving limiter[17], minmod limiter[8], or 
Krivodonova limiter[18]. In the present work we use maximum preserving limiter for concentration function in nonconservative transport equation (13) and Krivodonova moment limiter for hyperelastic model (1)-(3).

\section{NUMERICAL RESULTS}

The described algorithm was implemented as program code using $\mathrm{C}++$ language. The feature of software implementation is usage thermodynamical potential with its natural variables as EOS. For appropriate medium properties calculation the automatic differentiation technique is used (STAN[19]). Thermodynamical parameters (stress tensor, acoustic tensor, temperature, entropy, etc) are obtained directly from thermodynamical potential without numerical approximation of its derivatives. The developed program also uses libraries BOOST[20] and EIGEN[21].

In present section the numerical results for Godunov-Romenskii model are given for homogeneous and heterogeneous medium testcases. The well-known model tests for solid and gaseous phases are considered. In the examples below, initial value problems are solved in a computational domain $\Omega=[0,1] \mathrm{cm}$. The position of the discontinuity in the initial data is $x=0.5 \mathrm{~cm}$.

\subsection{Homogeneous case}

Gas dynamics. As mentioned above, Godunov-Romenskii model can describe gas flow assuming that EOS is chosen in a proper way. This approach is used here to solve the well-known Sod shock tube problem[22], adapted for hyperelastic model setting. Complete hyperelastic model with 13 equations for variables $\left(u_{k}, F_{i j}, \mathrm{~S}\right)$ is considered instead Euler ideal gas dynamics system with 5 equations[23].

Mesh step is $0.001 \mathrm{~cm}$. Time step is $0.01 \mathrm{sec}$. The piecewise polynomials inside each cell are up to third order. As it is mentioned above, EOS consists of only hydrodynamical term (9)

$$
\mathrm{U}=\mathrm{U}^{\mathrm{h}}\left(I_{3}, \mathrm{~S}\right)=c_{V} T_{0} I_{3}^{\gamma / 2}\left(\exp \left[\mathrm{S} / c_{V}\right]-1\right) .
$$

with $\rho_{0}=1.0 \mathrm{~g} / \mathrm{cm}^{3}$ being initial density, $c_{V}=1.0 \cdot 10^{-6} \mathrm{~kJ} /(\mathrm{gK})$ - heat capacity, $T_{0}=100 \mathrm{~K}$ - reference temperature, $b_{0}=0.0 \mathrm{~km} / \mathrm{s}-$ shear wave speed and $\gamma=0.4-$ constant parameters.

Distortion tensor coefficients and entropy values are chosen in such a way that they correspond to the parameters of the Sod problem for Euler equations. The initial state corresponds to Riemann problem with two constant states:

$$
\begin{aligned}
& \mathbf{u}_{1}=\left(\begin{array}{l}
0 \\
0 \\
0
\end{array}\right) \frac{\mathrm{km}}{\mathrm{s}}, \quad \mathbf{F}_{1}=\left(\begin{array}{lll}
1 & 0 & 0 \\
0 & 1 & 0 \\
0 & 0 & 1
\end{array}\right), \quad \mathrm{S}_{1}=4.0 \cdot 10^{-6} \frac{\mathrm{kJ}}{\mathrm{gK}}, \\
& \mathbf{u}_{\mathrm{r}}=\left(\begin{array}{l}
0 \\
0 \\
0
\end{array}\right) \frac{\mathrm{km}}{\mathrm{s}}, \quad \mathbf{F}_{\mathrm{r}}=\left(\begin{array}{lll}
8 & 0 & 0 \\
0 & 1 & 0 \\
0 & 0 & 1
\end{array}\right), \quad \mathrm{S}_{\mathrm{r}}=1.7 \cdot 10^{-6} \frac{\mathrm{kJ}}{\mathrm{gK}},
\end{aligned}
$$


where indices "l" and " $r$ " denotes left and right states, respectively.

Figure 1 shows various state dimensionless profiles at time $t=0.6 \mathrm{sec}$. The results are fully identical to gas dynamics ones[23].

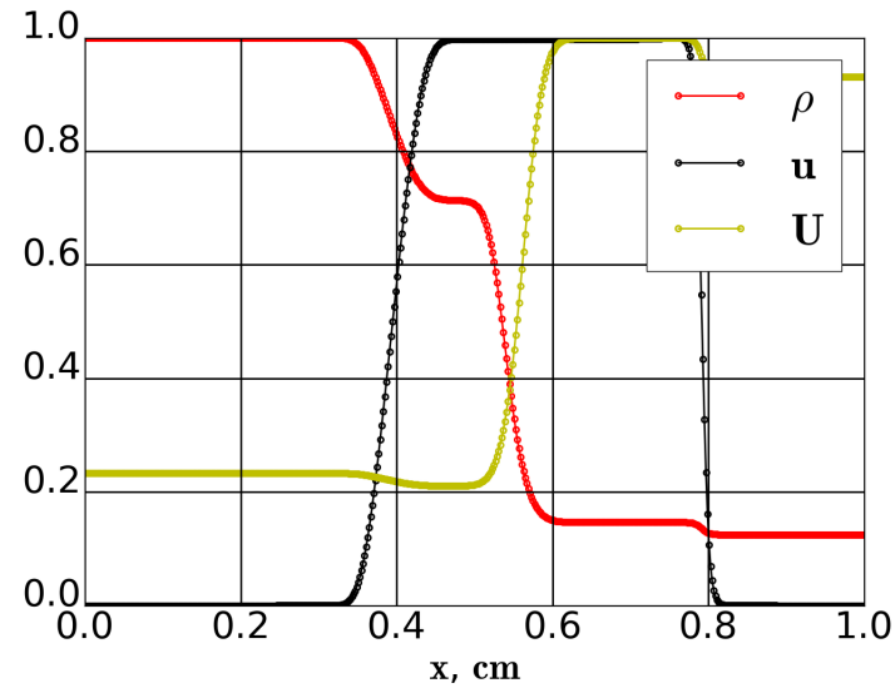

Figure 1: Dimensionless density $(\rho)$, velocity $(u)$ and internal energy ( $\mathrm{U}$ ) profiles at a time $t=0.6 \mathrm{~s}$.
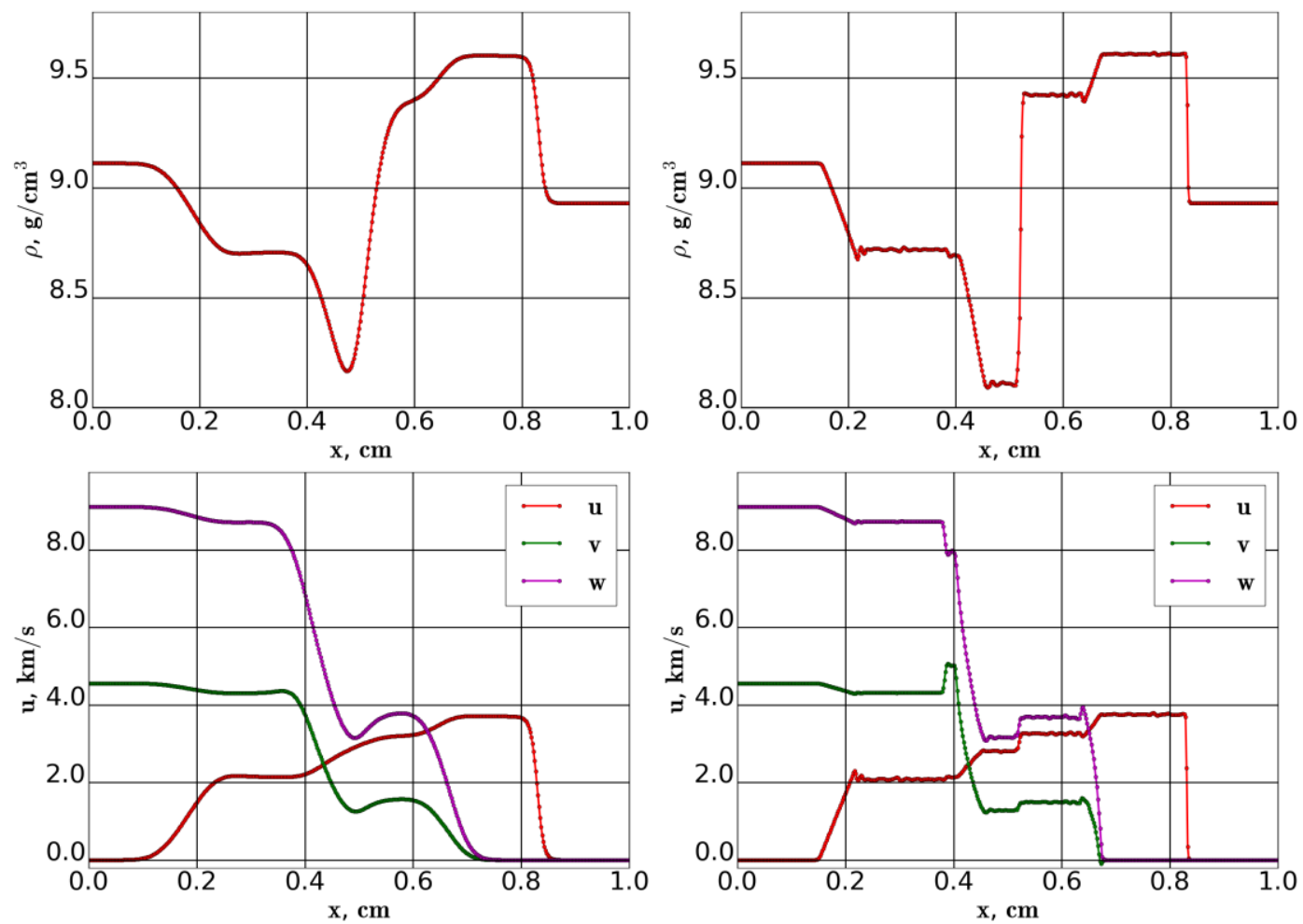

Figure 2: Density $\rho$ (up) and velocities $u, v, w$ (down) distributions for finite volume (left) and RK/DG (right) methods at time $0.5 \mu \mathrm{s}$.

Nonlinear hyperelasticity. This test is from [4] with nondiagonal distortion tensor. The $\mathrm{RK} / \mathrm{DG}$ and finite volume methods are considered. Mesh step is $0.002 \mathrm{~cm}$. Time step is 0.005 
$\mu \mathrm{s}$. Piecewise polynomials in each cell are considered up to third order. The initial state is given by:

$$
\begin{gathered}
\mathbf{u}_{1}=\left(\begin{array}{c}
0 \\
0.5 \\
1
\end{array}\right) \frac{\mathrm{km}}{\mathrm{s}}, \quad \mathbf{F}_{1}=\left(\begin{array}{ccc}
0.98 & 0 & 0 \\
0.02 & 1 & 0.1 \\
0 & 0 & 1
\end{array}\right), \quad \mathrm{S}_{1}=10^{-3} \frac{\mathrm{kJ}}{\mathrm{gK}}, \\
\mathbf{u}_{\mathrm{r}}=\left(\begin{array}{l}
0 \\
0 \\
0
\end{array}\right) \frac{\mathrm{km}}{\mathrm{s}}, \quad \mathbf{F}_{\mathrm{r}}=\left(\begin{array}{ccc}
1 & 0 & 0 \\
0 & 1 & 0.1 \\
0 & 0 & 1
\end{array}\right), \quad \mathrm{S}_{\mathrm{r}}=0 \frac{\mathrm{kJ}}{\mathrm{gK}} .
\end{gathered}
$$

The material is assumed to be copper with EOS parameters defined in [4]: $\rho_{0}=8.9 \mathrm{~g} / \mathrm{cm}^{3}$, $c_{0}=4.6 \mathrm{~km} / \mathrm{s}, c_{V}=3.9 \cdot 10^{-4} \mathrm{~kJ} /(\mathrm{g} \cdot \mathrm{K}), T_{0}=300 \mathrm{~K}, b_{0}=2.1 \mathrm{~km} / \mathrm{s}, \alpha=1.0, \beta=3.0, \gamma=2.0$.

Results are shown in Figure 2 at time $0.5 \mu \mathrm{s}$ in the comparison with finite volume method results. RK/DG method has better resolution of waves. The results are fully identical to the published ones[4].

\subsection{Heterogeneous case}

In this testcase mesh step is $h=5 \cdot 10^{-4} \mathrm{~cm}$ and time step is $0.005 \mu \mathrm{s}$. The polynomials inside each cell are considered up to third order.

Consider the heterogeneous medium model described in subsection 2.1. Homogeneous domains correspond to $\Omega_{1}=(0,1 / 2]$ and $\Omega_{\mathrm{r}}=(1 / 2,1)$. The smoothed characteristic functions for $\Omega_{1, \mathrm{r}}$ are chosen as $\phi_{1}=1-\phi_{\mathrm{r}}$,

$$
\phi_{1}=\left\{\begin{array}{cc}
0, & x \in(0,1 / 2-\varepsilon) \\
\frac{1}{2}-\frac{1}{2 \varepsilon}\left(x-\frac{1}{2}\right)-\frac{1}{2 \pi} \sin \left(\frac{\pi}{\varepsilon}\left(x-\frac{1}{2}\right)\right), & x \in[1 / 2-\varepsilon, 1 / 2+\varepsilon] \\
1, & x \in(1 / 2+\varepsilon, 1),
\end{array}\right.
$$

where $\varepsilon=10 \mathrm{~h}$. Initial conditions are given by[4]:

$$
\begin{aligned}
& \mathbf{u}_{1}=\left(\begin{array}{c}
2 \\
0 \\
0.1
\end{array}\right) \frac{\mathrm{km}}{\mathrm{s}}, \quad \mathbf{F}_{1}=\left(\begin{array}{ccc}
1 & 0 & 0 \\
-0.01 & 0.95 & 0.02 \\
-0.015 & 0 & 0.9
\end{array}\right), \quad \mathrm{S}_{1}=0 \frac{\mathrm{kJ}}{\mathrm{gK}}, \\
& \mathbf{u}_{\mathrm{r}}=\left(\begin{array}{c}
0 \\
-0.03 \\
-0.01
\end{array}\right) \frac{\mathrm{km}}{\mathrm{s}}, \quad \mathbf{F}_{\mathrm{r}}=\left(\begin{array}{ccc}
1 & 0 & 0 \\
0.015 & 0.95 & 0 \\
-0.01 & 0 & 0.9
\end{array}\right), \quad \mathrm{S}_{\mathrm{r}}=0 \frac{\mathrm{kJ}}{\mathrm{gK}} .
\end{aligned}
$$

Left material EOS parameters: $\rho_{0}=8.93 \mathrm{~g} / \mathrm{cm}^{3}, c_{0}=4.6 \mathrm{~km} / \mathrm{s}, \quad c_{V}=3.9 \cdot 10^{-4} \mathrm{~kJ} /(\mathrm{g} \cdot \mathrm{K})$, $T_{0}=300 \mathrm{~K}, b_{0}=2.1 \mathrm{~km} / \mathrm{s}, \alpha=1.0, \beta=3.0, \gamma=2.0$. 

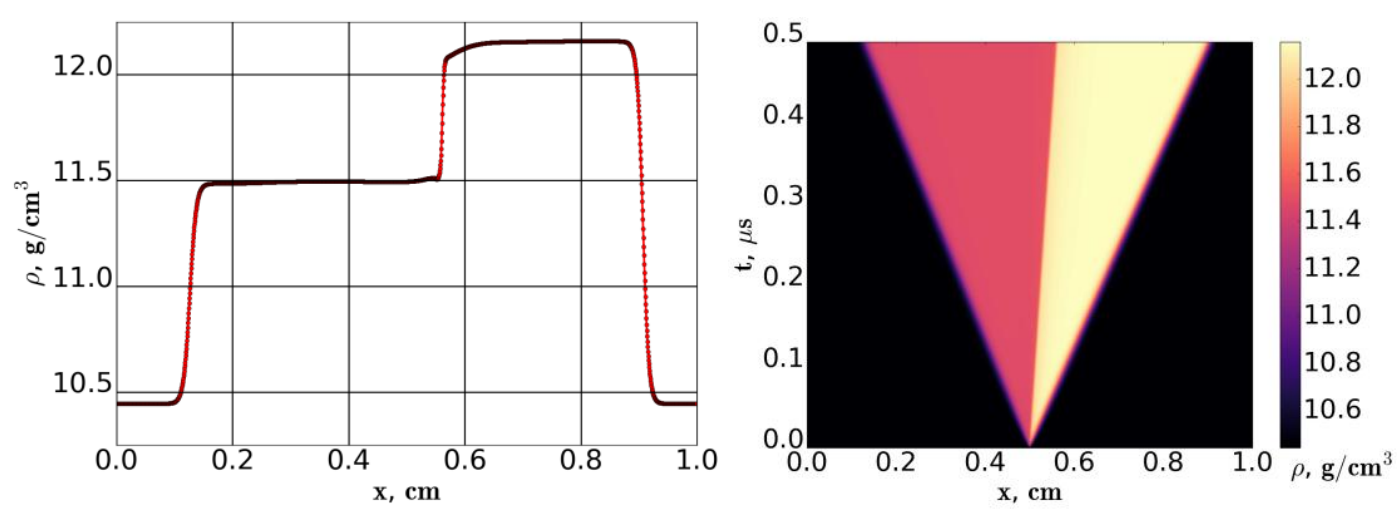

Figure 3: Denstiy ( $\rho$ ) distribution at $t=0.5 \mu \mathrm{s}$ (left) and $x$ - $t$ diagram (right).
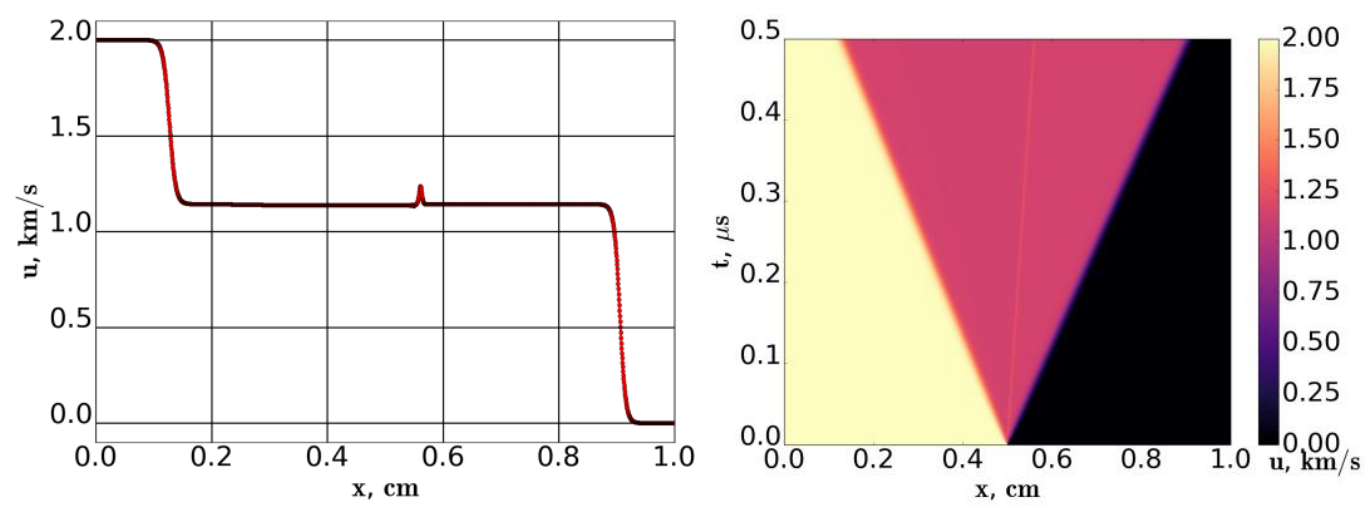

Figure 4: Velocity ( $u$ ) distribution at $t=0.5 \mu \mathrm{s}$ (left) and $x$ - $t$ diagram (right).
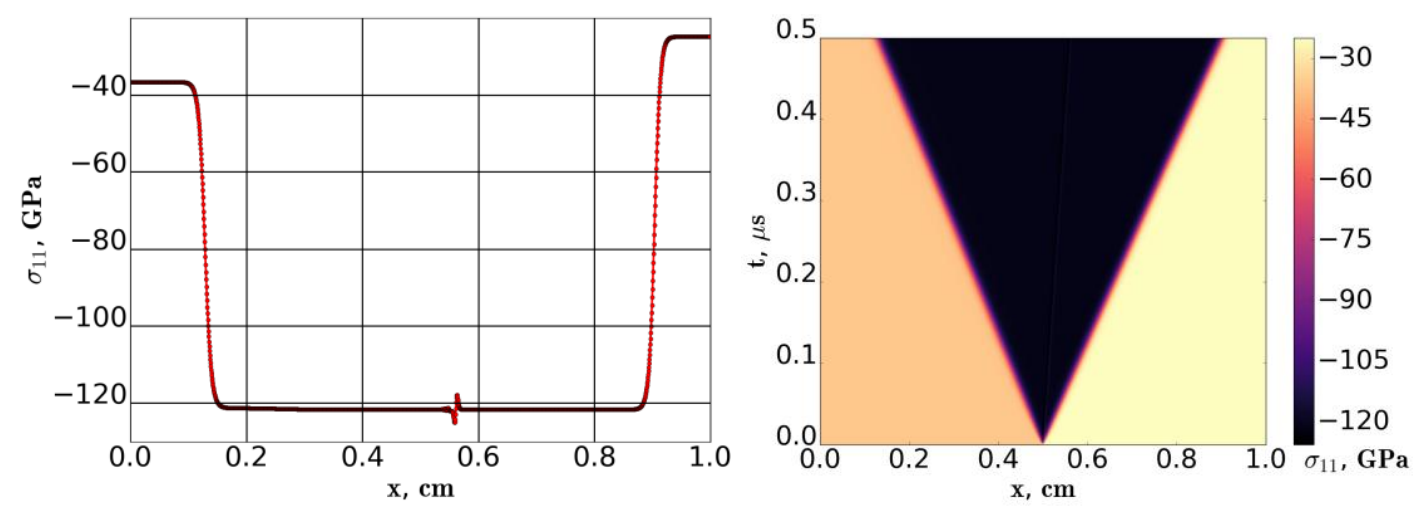

Figure 5: Stress tensor component ( $\left.\sigma_{11}\right)$ distribution at $t=0.5 \mu \mathrm{s}$ (left) and $x$ - $t$ diagram (right).

Right material EOS parameters: $\rho_{0}=8.93 \mathrm{~g} / \mathrm{cm}^{3}, c_{0}=6.22 \mathrm{~km} / \mathrm{s}, c_{V}=9.0 \cdot 10^{-4} \mathrm{~kJ} /(\mathrm{g} \cdot \mathrm{K})$, $T_{0}=300 \mathrm{~K}, b_{0}=3.16 \mathrm{~km} / \mathrm{s}, \alpha=1.0, \beta=3.577, \gamma=2.088$.

Solution at time $t=0.5 \mu \mathrm{s}$ is shown in Figures 3-5. Interphase boundary is moving from left to right. Left figures correspond to variables profiles at given time, right ones are corresponding $x$ - $t$ diagrams. 
In Figure 3 the density profile and corresponding $x-t$ diagram is shown at time $t=0.5 \mu \mathrm{s}$. In Figures 4 and 5 velocity $u$ and stress tensor component $\sigma_{11}$ and corresponding $x-t$ diagrams are shown.

\section{CONCLUSION}

The paper discusses the application of the RK/DG method for solving problems of hyperelasticity in an inhomogeneous medium. Both models, the homogeneous and heterogeneous one, admitting piecewise-constant distribution of medium properties, are investigated. As a result of a series of calculations, it was shown that the Godunov-Romenskii hyperelastic model can be practically applied to solve gas dynamics problems, when the internal energy of a medium depends only on its bulk deformations and entropy. The application of the RK/DG method demonstrates sharp resolution of wavefronts, comparable to the use of methods of the WENO type.

\section{REFERENCES}

[1] Godunov S.K., Romenskii E.I., "Nonstationary equations of nonlinear elasticity theory in Eulerian coordinates", J. Appl. Mech. Tech. Phys., 13(6), 868-884 (1972).

[2] Favrie N., Gavrilyuk S.L., Saurel R., "Solid-fluid diffuse interface model in cases of extreme deformations", J. Comput. Phys., 228(16), 6037-6077 (2009).

[3] Saurel R., Petitpas F., Berry R.A., "Simple and efficient relaxation methods for interfaces separating compressible fluids, cavitating flows and shocks in multiphase mixtures", J. Comput. Phys., 228(5), 1678-1712 (2009).

[4] Barton P.T. et al., "Exact and approximate solutions of Riemann problems in non-linear elasticity", J. Comput. Phys., 228(18), 7046-7068 (2009).

[5] Miller G.H., "Minimal rotationally invariant bases for hyperelasticity", SIAM J APPL MATH, 64(6), 2050-2075 (2004).

[6] Liu X.D., Osher S., Chan T., "Weighted essentially non-oscillatory schemess", J. Comput. Phys., 115(1), 200-212 (1994).

[7] Jiang G.S., Shu C.W., "Efficient implementation of weighted ENO schemes", J. Comput. Phys., 126(1), 202-228 (1996).

[8] Cockburn B., Shu C.W., "The Runge-Kutta local projection discontinuous-Galerkin finite element method for scalar conservation laws", ESAIM-MATH MODEL NUM, 25(3), 337-361 (1991).

[9] Baer, M.R., Nunziato, J.W., "A two-phase mixture theory for the deflagration-to-detonation transition (DDT) in reactive granular materials", Int. J. Muhiphase Flow, 12(6), 861-889 (1986).

[10] Godunov S. K., Romenskii E., Elements of continuum mechanics and conservation laws, Springer Science and Business Media (2003).

[11] Dimitrienko Y.I., Nonlinear continuum mechanics and large inelastic deformations, Springer Science and Business Media, Vol.174., (2010).

[12] Kapilla, A.K., Menikoff, R., Bdzil, J.B. et al., "Two-phase modelling of deflagration to detonation transition in granular materials: reduced equations", PHYS FLUIDS, 13(10), 3002-3024 (2001).

[13] Dal Maso G., Le Floch P., Murat F., "Definition and weak stability of nonconservative products", J. Math. Pures Appl, 74(6), 483-548 (1995).

[14] Kolmogorov A. N., Fomin S. V., Elements of the theory of functions and functional analysis, Vol. 1., (1957).

[15] Michoski C. et al., "A comparison of artificial viscosity, limiters, and filters, for high order discontinuous Galerkin solutions in nonlinear settings", J. Sci. Comput., 66(1), 406-434 (2016).

[16] Persson P.O., Peraire J., "Sub-cell shock capturing for discontinuous Galerkin methods", 44th 
AIAA Aerospace Sciences Meeting and Exhibit, 112 (2006).

[17] X. Zhang, Y. Xia, C.-W. Shu., "Maximum-principle-satisfying and positivity-preserving high order discontinuous Galerkin schemes for conservation laws on triangular meshes", J. Sci. Comput., 50(1), 29-62 (2012).

[18] Krivodonova L., "Limiters for high-order discontinuous Galerkin methods", J. Comput. Phys., 226(1), 879-896 (2007).

[19] The Stan Math Library. https://github.com/stan-dev/math (Accessed January 19, 2020).

[20] BOOST C++ libraries. https://www.boost.org/ (Accessed January 10, 2020).

[21] EIGEN. http://eigen.tuxfamily.org/index.php?title=Main Page (Accessed January 5, 2020).

[22] Sod G.A., "A survey of several finite difference methods for systems of nonlinear hyperbolic conservation laws", J. Comput. Phys., 27(1), 1-31 (1978).

[23] Toro E.F., Riemann solvers and numerical methods for fluid dynamics: a practical introduction, Springer Science and Business Media (2013).

[24] Egorova V.A., Uskov R.V., Voronin F.N., Zhukovskiy M.E., "On the modeling of the thermomechanical fields in porous material being under radiation", Mathematica Montisnigri, 43, 58-69 (2018).

[25] Markov M.B., Uskov R.V., Zhukovskiy M.E., "Monte-Carlo modeling of the photon-electron cascade In heterogeneous matter", Mathematica Montisnigri, 46, 49-60 (2019).

Received January 10, 2020 